\title{
A Ffowcs Williams-Hawkings numerical aeroacoustic study of varied and fixed-pitch blades of an H-Rotor vertical axis wind turbine
}

\author{
Errol Molatudi ${ }^{1, *}$, Thokozani Justin Kunene ${ }^{1}$, and Lagouge Kwanda Tartibu ${ }^{1}$ \\ ${ }^{1}$ University of Johannesburg, Mechanical \& Industrial Engineering Technology Department, \\ Doornfontein Campus, Johannesburg, South Africa
}

\begin{abstract}
The effects of sound pressure level at two observation positions of a fixed and varied blade pitch angle at Low-Mach unsteady incompressible Reynolds-Average Navier-Stokes flow approach, on an H-rotor Vertical Axis Wind Turbine. The objective of the study is to compare the noise dissipation and output power/energy of the airfoil blades design of the vertical axis wind turbine in residential zones. The Ffowcs Williams -Hawkings (FHWH) techniques were applied to validate the output noise and vortex shedding of the different angles of attacks (AoA). The study postulated that the time history of the calculated sound pressure level at two observers positions: the aeroacoustic, blade vortex interaction noise, flow separations, dynamic stall experience from varied angled of attacks are found to produces less noise and vortex shedding compared to the fixed angle of attack.
\end{abstract}

Keywords:

Aeroacoustic, Angle-of-Attack, Ffowcs-Hawkings, Sound Pressure level (SPL)

\section{Introduction}

There is a need for renewable energy globally, and wind energy is another alternative that can provide clean energy to residential areas. Wind energy has shown great potential since 2006 in China. China is currently leading in wind energy development, with a cumulative installed capacity of wind power increase from 23,900 MW in 2001 to 651,000 MW in 2019 [1]. A wind turbine is an attractive form of electricity production for residential households. However, the wind harnessing energy process comes with excellent noise pollution. The noise generated by the operating wind turbine significantly impacts surrounding residential areas, particularly humans and animals, respectively. Mechanical noise and aerodynamic noise are two types of noise produced by wind turbines. Moving machinery parts, gearboxes, and generators are common sources of mechanical noise [2]. Aerodynamic noise can be narrow-band or broadband, depending on the rotor geometry, blade geometry, and flow environment [3]. Because there are two types of wind turbines that can create electricity for residents,

\footnotetext{
*Errol Molatudi molatudierrol@gmail.com
} 
A vertical axis wind turbine is preferred over a horizontal axis wind turbine because it offers greater benefits. The noise pollution produced by a vertical axis wind turbine is lower than that produced by a horizontal axis wind turbine [4]. To obtain less aerodynamic, the turbine must run at a low-speed ratio range and a higher angle of attack[5]. Reduced noise pollution is dependent not only on the angle of attack, but also on how the wind interacts with the blades, known as vorticity; research has shown that minimizing vorticity with the blade can result in improved turbine performance [6] The different trailing edges, tip forms, and airfoil profiles have all been studied in order to reduce aerodynamic noise from wind turbines. The airflow speed around the blades, which is related to the rotor tip-speed ratio, is an important contributor to noise emission. As a result of these discoveries, several blade angles of attack have been developed, including fixed blades and swivel blades with a fixed angle of attack. [7].

A $5 \mathrm{~kW}$ semi-scale three-blade H-rotor vertical axis wind turbine with a fixed and variable angle of attack will be investigated in this study. The flow dynamics of a Darrieus H-rotor three-blade vertical axis turbine are significantly influenced. The airfoils are controlled by the leading edge slats and flaps, which increase cambering and improve flight or flow via lifting characteristics. The impacts of blade form, speed ratio, solidity, and distance between noise sources and receivers would be determined using an Ffowc William-Hawking model. Because the Ffowcs William-Hawking is concerned with rotating zones and sliding mesh models. In ANSYS-FLUENT, Ffowcs Willam - Hawking models have been implemented. As a result, all of the findings were obtained using the commercial CFD program ANSYSFLUENT 2019 R1. The time-accurate solutions are obtained using an Unsteady Reynoldsaveraged Navier-Stokes model [8]. H-rotor vertical axis wind turbines, also known as Darrieus vertical axis wind turbines, have been the subject of a lot of research since they produce a lot of electricity with little noise pollution [9].

\section{Noise pollution}

A wind turbine produces two sorts of noise when it is in operation: mechanical and aerodynamic noise. Machinery parts such as gearboxes and generators generate mechanical noise. It could be effectively reduced using a well-known engineering design [2]. Aerodynamic noise, on the other hand, remains a challenging task to predict. VAWT noise sources can be categorised. The high-frequency noise created by an airfoil's interaction with flow disturbances produced in its boundary layer and near wake is known as airfoil self-noise. Airfoil self-noise occurs in a vertical axis wind turbine when excess turbulence interacts with the blade surface, causing Stall/Separation noise. Interaction causes tip vortex noise. Theoretical concepts are typically utilized to forecast an airfoil's noise, whereas experimentally generated components are employed to better agree with what is observed in practice. Serrated trailing edges, various trailing edge and tip forms, and various airfoil shapes have all been researched to reduce wind turbine aerodynamic noise.

\section{Acoustics model theory}

\subsection{The Ffows Williams Hawkings model}

The same continuity and Navier stokes equations are used to derive the Ffows Williams Hawkings equation. The Ffowcs Williams technique, which assumes that the detailed pressure and velocity fluctuations are known, is frequently used to enhance turbine noise 
prediction. Ffowcs William and Farassat derived the genesis of this wave equation, propeller and helicopter rotor noise. The Ffowcs William and Hawkings equation is an inhomogeneous wave equation that includes the effects of moving surfaces. When the surface is in the near field of a solid body, the FW-H technique filters out the part of the solution that does not radiate as sound. [10].The key to determining any rotor noise applying Ffowcs William Hawking method, there are three terms of importance [11] The sound radiated by turbulence and flow distortion. A dipole is controlled by blade surface loading. The motion of the source compresses the time history as the source moves towards the observer, thus an increase in radiated sound.

The Ffowcs Williams Hawking equation helps to calculate noise signals. There have been improvements in determining the varying flow of practical interest. The only limit would be the size of the computer to generate unsteady flow and acoustics inside a computational grid, provided the Mach number is not too high. The linear wave equation can model the wave propagation outside the flow, so increasing the size of the computational domain is wasteful. The Ffowcs William Hawking will provide solutions to wave equations, given that the numerical calculations are accurate on a surface that bounds the source and that compressible flow fluctuation and the acoustic waves are captured accurately. [12] The frequency-domain version is totally dependant on the equation developed by Lockard. The Ffowcs William Hawking uses the equation s for two-dimensional problems, the derivation of this equation a Cartesian tensor notation was employed which is equally valid in three-dimensions. At the location of each receiver is predicted from small amplitude acoustic pressure fluctuations time-accurate solutions of the flow field variable around wind turbine are required.

Ffowcs Williams Hawkings' acoustic analogy is developed for simulating the moving surface acoustic radiation in the far-field. The Ffowcs Williams Hawkings is developed from the Navier-Stokes equations. Mohamed et al [8] represent the FHWH equations as:

$$
\begin{gathered}
\frac{1}{a_{o}^{2}} \frac{\partial^{2} \dot{p}}{\partial t^{2}}-\nabla^{2} \dot{p}=\frac{\partial^{2}}{\partial x_{i} \partial x_{j}}\left\{T_{i j} H(f)\right\}-\frac{\partial}{\partial x_{i}}\left\{\left[P_{i j} n_{j}+\rho u_{i}\left(u_{n}-v_{n}\right)\right]\right. \\
\delta(f)\}+\frac{\partial}{\partial t}\left\{\left[\rho_{o} v_{n}+\rho\left(u_{n}-v_{n}\right)\right] \delta(f)\right\}
\end{gathered}
$$

Where :

$u_{i} \quad=$ Fluid velocity component in the $x_{i}$ direction

$u_{n} \quad=$ Surface velocity component normal to the surface $f=0$

$v_{i} \quad=$ Surface velocity components in the $x_{i}$ direction

$v_{n} \quad=$ Surface velocity component normal to the surface

$\partial(f)=$ Dirac delta function

$H(f)=$ Heaviside function

$\dot{p} \quad=$ The sound pressure at the far-field, $\not \dot{p}=\left(P-P_{0}\right)$

$T_{i j} \quad=$ The Lighthill's stress tensor, $T_{i j}=\rho u_{i} u_{j}+P_{i j}-a_{o}^{2}\left(\rho-\rho_{o}\right) \delta_{i j}$

$a_{0}^{2} \quad=$ The speed of sound

$P_{i j} \quad=$ The compressive stress tensor

Where the compressive stress tensor can be further broken:

$P_{i j}=p \delta_{i j}-\mu\left[\frac{\partial u_{i}}{\partial x_{j}}+\frac{\partial u_{j}}{\partial x_{i}}-\frac{2}{3} \frac{\partial u_{k}}{\partial x_{k}} \delta_{i j}\right]$ 
A green function is used to compute the sound pressure at the receiver location. The subscript o denotes Free-stream quantities. The free-space Green function $(\delta(\mathrm{g}) / 4 \pi \mathrm{r})$ is used to find the solution to Eq. (1). The entire solution is made up of surface and volume integrals. The surface integrals explain the contributions from monopole and dipole acoustic sources and specific contributions from quadrupole sources, whereas the volume integrals describe quadrupole (volume) sources outside the source surface. When the flow is below subsonic, as in the Darrieus turbine instance, the contribution of the volume integrals becomes minor. Volume integrals are not used in ANSYS-FLUENT 2019 R1 [8]. As a result:

$p(\vec{x}, t)=\dot{p}_{T}(\vec{x}, t)+\dot{p}_{L}(\vec{x}, t)$

Where:

$$
\begin{aligned}
4 \pi \dot{p}_{T}(\vec{x}, t)= & \int_{f=0}\left[\frac{\rho_{o}\left(\dot{U}_{n}+U_{\dot{n}}\right)}{r\left(1-M_{r}\right)^{2}}\right] \mathrm{d} s+\int_{f=0}\left[\frac{\rho_{o} U_{n}\left\{r \dot{M}_{r}+a_{o}\left(M_{r}-M^{2}\right)\right\}}{r^{2}\left(1-M_{r}\right)^{3}}\right] \mathrm{d} s \\
4 \pi \dot{p}_{L}(\vec{x}, t)= & \frac{1}{a_{0}} \int_{f=0}\left[\frac{\dot{L_{r}}}{r\left(1-M_{r}\right)^{2}}\right] \mathrm{d} s+\int_{f=0}\left[\frac{L_{r}-L_{M}}{r^{2}\left(1-M_{r}\right)^{2}}\right] \mathrm{d} s+ \\
& \frac{1}{a_{0}} \int_{f=0}\left[\frac{L_{r}\left\{r \dot{M}_{r}+a_{o}\left(M_{r}-M^{2}\right)\right\}}{r^{2}\left(1-M_{r}\right)^{3}}\right] \mathrm{d} s
\end{aligned}
$$

Where:

$$
\begin{aligned}
& U_{i}=v_{i}+\frac{\rho}{\rho_{o}}\left(u_{i}-v_{i}\right) \\
& L_{i}=P_{i j} \hat{n}_{j}+\rho u_{i}\left(u_{n}-v_{n}\right)
\end{aligned}
$$

With the integration surface synchronizing with an impenetrable wall, the two components on the right in Eq. (3), $\dot{p}_{T}(\vec{x}, t)$ and $p_{L}(\vec{x}, t)$ are sometimes referred to as thickness and loading terms, respectively, due to their physical definitions. In Eqs. (4) and (5), the square brackets denote that the kernels of the integrals are computed at the corresponding retarded times, which are defined as follows, given the observer time, $t$, and distance to the observer, r.

$\tau=t-\frac{r}{a_{o}}$

The different subscripted numbers in Eqs. (6) and (7) are the inner products of a vector and a unit vector, which are made tacit by the subscript. For example, $L_{r}=\vec{L} \cdot \vec{r}=L_{i} r_{i}$ and $L_{r}=$ $\vec{U} \cdot \vec{n}=U_{i} n_{i}$, where $\vec{r}$, and $\vec{n}$ represent the unit vectors in the radiation and wall normal directions, respectively. The dot over a variable indicates that variable's source-time differentiation [8]. There are a few comments about the applicability of this integral solution:

a. The FW-H formulation can be used to treat both revolving and stationary surfaces.

b. It is not necessary for the surface $f=0$ to coincide with body surfaces or walls. Because the formulation makes source surfaces permeable, they can be positioned in the flow's interior.

c. When a permeable source surface (either an interior or non-conformal sliding interface) is positioned at a specific distance from the body surface, the integral solutions obtained by Eqs. (5) and (6) include contributions from quadrupole sources within the source surface's region. The mesh resolution must be fine enough to resolve the 
transient flow structures inside the volume contained by the permeable source surface when utilizing a permeable source surface.

\section{Numerical model}

\subsection{Geometry and topology of VAW}

The three-blade configuration is on the assumption that the three blades are more stable in comparison to the two or four-blade configuration(see Fig: 1) [13]., furthermore research is conducted on the aspect on a vertical axis wind turbine, that a higher aspect ratio has a lower power coefficient and vice versa [14] [15].

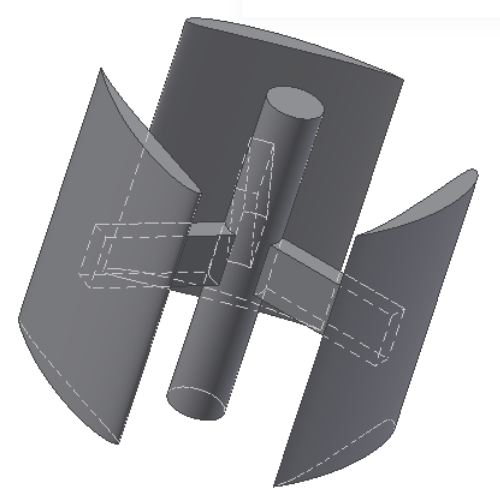

(a)

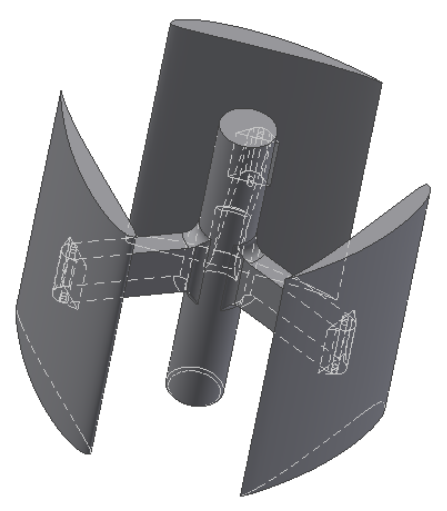

(b)

Fig. 1. Three-blade H-type VAWT, rigid (fixed AOA) and (b) swivel (varied AoA, sinusoidal profiled),

\subsection{Mesh and grid sensitivity}

The mesh was generated using the Body-influence method (see Fig: 2) to refine local bodies, i.e., the rotating core that houses the blades. The stationary body around is coarsened, but the rotating core is refined. The interface between the rotating core and the stationary body was included in the refinement of the mesh. The first layer of $1 \times 10^{-5} \mathrm{~m}$ from 12 prismatic layers leading up to the wall plus value of, $\mathrm{y}^{+}=5$. The limit of dynamic stall of blade Reynolds number, $\mathrm{Re}_{\mathrm{b}}=771707$ at an azimuth angle, $\theta=1.5^{\circ}$ of the blades (or rotating core in this case) when the rotation reaches 1 second.

- $\quad \mathrm{Re}_{\mathrm{b}}$ is found on a fixed tip speed ratio (TSR), of 0.56.

- This TSR places the blades out of the static stall [15]. The modelled blades have a maximum stall angle of 0.9620 . 

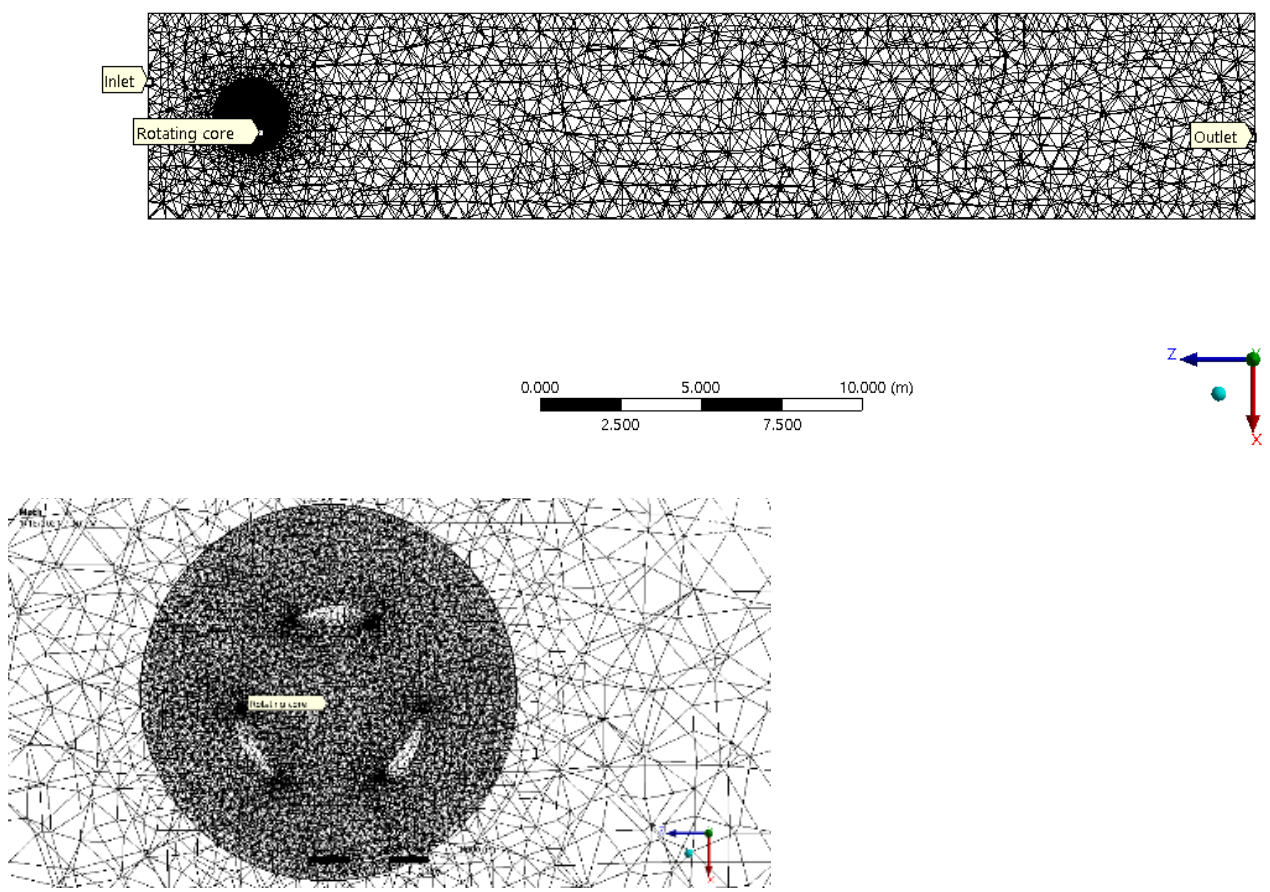

Fig. 2. Mesh density of a far-field computational domain, tetrahedral elements

\subsection{Computational domain and boundary conditions}

As published, the velocity inlet of $15 \mathrm{~m} / \mathrm{s}$ is a wind speed around residential areas [16], [17]. We modelled a 10\% turbulence intensity at the inlet (upstream) of the rotating core. The rotating core rotates the blades at an angular speed of, $\Omega=15 \mathrm{rad} / \mathrm{sec}$, based on the Tip Speed Ratio (TSR) of 0.56 .

\subsection{Solver and turbulence model}

Unsteady incompressible Reynolds-averaged The commercial CFD code ANSYS Fluent 2019 R1 was used to solve Navier-Stokes (URANS) simulations. Allmaras, Spalart (S-A model). Kostic [18] articulates the SA one-dimensional equations effectively. The impacts of blade form, speed ratio, solidity, and distance between noise sources and receivers would be determined using an Ffowc William-Hawking model. Since the Ffowcs William-Hawking is concerned with rotating zones and a sliding mesh model. As this Ffowcs Willam -Hawking models have been built in ANSYS-FLUENT, all results are calculated using a commercial CFD program ANSYS-FLUENT. The time accurate solutions are obtained using an Unsteady Reynolds-averaged Navier-Stokes model [8]. Many studies on the H-rotor vertical axis wind turbine, also known as the Darrieus Vertical axis wind turbine, have revealed high energy production with reduced noise pollution. [9]. For pressure-velocity coupling, a SIMPLE system is utilized. The spatial discretization utilized was a second-order accurate Finite Volume Method (FVM) [15]. To capture sources that are time-dependent turbulent quantities, such as the vortex expanding behind the aerofoils, a transient solution was used. 
The convergence criterion for all residuals was set to $1 \times 10^{9}$ for all governing equations. The temporal discretization of this unstable flow problem necessitated a solution in which a dynamic condition rotated blades and extracted energy from the incoming stream [19]. A Dynamic Mesh and an adapted mesh to two cells around each blade were employed for that actuation. It was anticipated that some mesh cells will exhibit negative meshing. The parameters that were affected included a time step $(t)$ that was set to the time required to complete an azimuthal increase $\left(d \theta==1 \times 10^{-5}\right)$ degrees, blade rotation, $\Delta t=1 \times 10^{-5} \mathrm{~s}$ with $1 \times 10^{+5}$ iterations [15]. For the interpreted six degree freedom function of all three blades rotating and a compiled UDF (centre of gravity, C G Motion for rotation) of sinusoidal pitching of the blades, two different User-Defined-Functions (UDF) were utilized (see Appendices A and B) (ANSYS Release 14, 2011). The shifting pitch angles ( $\beta$, Angles-ofAttack) of the blades, which are a bearing between the rotor arm and the blade, are assimilated by this UDF.

\subsection{Model validation}

For all the available and probable investigations around VAWT and aerofoils in general, there are still few 3D simulations. Even without the rotor arms, the 3D models provide a degree of freedom that occurs in reality, accounting for the flow moving in an axial vertical orientation. Figure 3 shows the distribution of the three observer positions (observer time positions) investigated for this study. At this time of reporting, the lack of experimental data for the current study about acoustic noise is only validated using similar CFD aeroacoustics studies found in the literature. Figure 4 validates this current study with the results obtained by $\mathrm{Su}$ et al. [4] for the three-bladed H-rotor VAWT turbine. The sound pressure level the observer positions from our study have a similar trend and magnitudes of the harmonics of the literature findings of $\mathrm{Su}$ et al. [4]. This work validates the zero angle-of-attack model. We continued to find results of the varied angle-of-angle, $\beta$. 


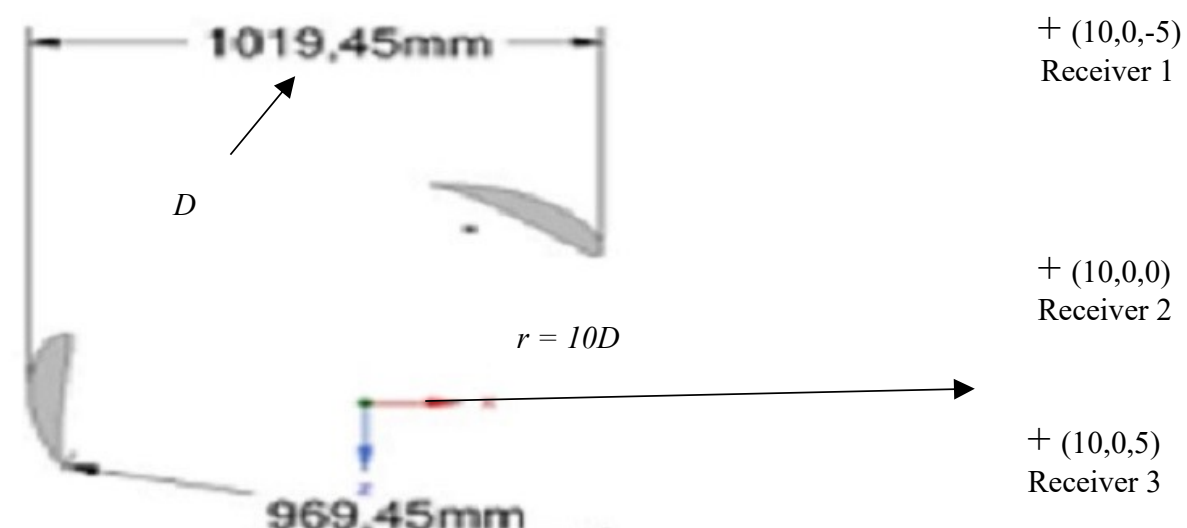

Fig. 3. The three prescribed observer positions at Cartisin coordinates (unit: $\mathrm{m}$ )

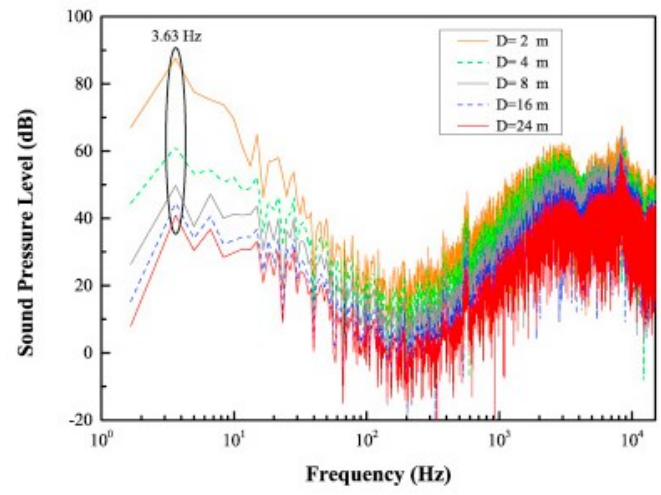




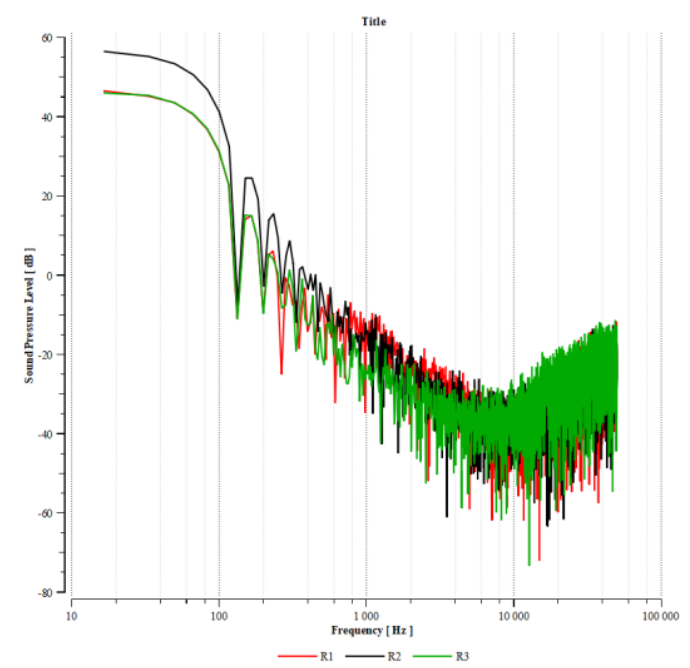

Fig. 4. Sound pressure level spectra at the prescribed observer positions, (a) Three-bladed H-rotor Darrieus turbine by Su et al $[4] \beta=0$, AoA, $\lambda=0.95$ at fcousing on $\mathrm{D}=8 \mathrm{~m}$ for the recivers (b) current CFD study at $\beta=0$, AoA, $\lambda=0.56$ at $\mathrm{D}=10 \mathrm{~m}$ for the recivers.

\section{Results and discussion}

Figure 5a shows that the blades are more potent in vortex sheets forming downstream and travelling further on a fixed angle of attack (AoA). However, the varied AoA to lower the stall separation as since with fewer vortex sheets being formed (Figure $5 \mathrm{~b}$ ). Therefore, the vortex sheet from varied AoA is quieter too. The varied AoA reduces flow separation and weakening the instability that periodically generates separation vortices, which leads to the vibration of rotating machinery and noise generation. We found the power coefficient $\left(\mathrm{C}_{\mathrm{p}}\right)$ in VAWT is maximum at low Tip-Speed-Ratio value $(\lambda=0.56)$. The results demonstrate that an SG6043 aerofoil blade with a chord length of $c=0.5 \mathrm{~m}$ captures the most energy in the presence of varying or fixed angle-of-attack.

\section{ANSYS}

ACADEMIC

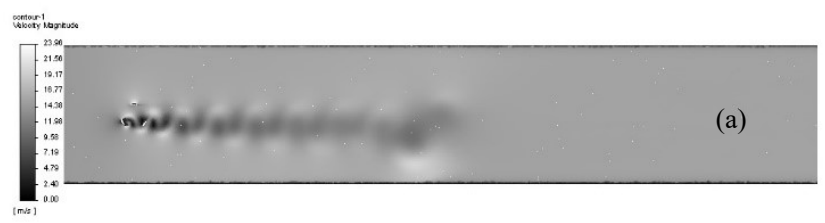




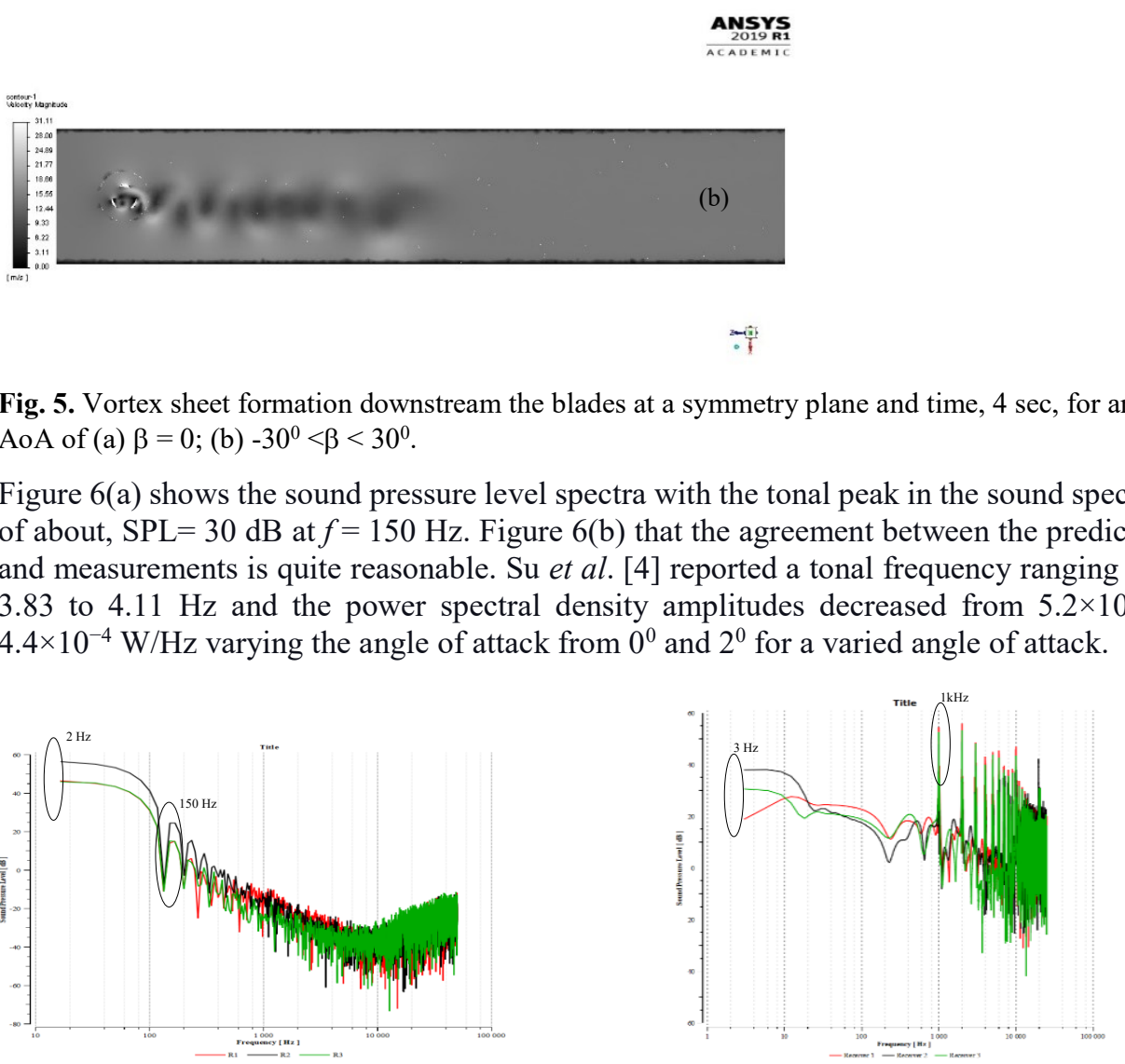

Fig. 6. Sound pressure level spectra AoA of (a) $\beta=0$; (b) $-30^{0}<\beta<30^{0}$ at three receiver positions
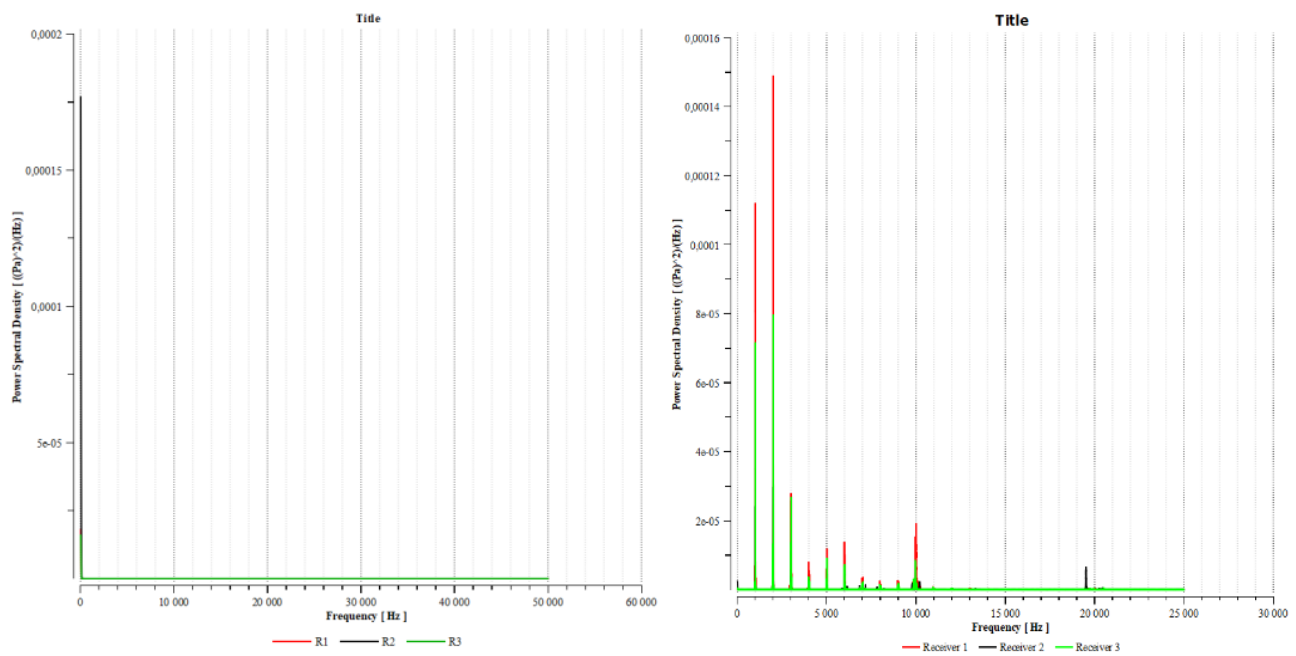

Fig. 7. Power spectra density AoA of (a) $\beta=0$; (b) $-30^{0}<\beta<30^{0}$ at three receiver positions 
Figure 7 is the power spectra density (PSD) of the current study as measured by the sound pressure level at observer positions. The sound emitted by the source is referred to as sound emission. The most essential quality, however, is noise immission [8], which relates to the sound perceived by an observer. There is a similar obversion with $\mathrm{Su}$ et al.[4] relating to the increase in tonal frequency and decrease in the power spectra density. Our results show a increase in the SPL at frequencies of $150 \mathrm{~Hz}$ and $1 \mathrm{kHz}$ at delayed higer frequencies for amplitudes. Amounting to a decrease in the PSD amplitudes from $1.75 \times 10^{-4}$ to $1.5 \times 10^{-4}$ $\mathrm{W} / \mathrm{Hz}$ (overall from all the receivers), respectively for fixed and varied AoA.

\section{Conclusion}

The aeroacoustic spectra of the fixed and varied angle of attack were simulated and analyzed: for a vertical-axis wind turbine with blades derived from a static tip speed ratio of 0.56 . This investigation has aided in the understanding of noise sources and aerodynamics responsible for aeroacoustic and improved power generation in vertical-axis wind turbines for an urban area with blades that vary their angle of attack as the turbine rotates.

The key findings of aerodynamics and aeroacoustics are summarized as follows: (1) The numerical investigation of a varied angle of attack and fixed angle of attack on a vertical axis wind turbine. The research postulates that the varied angle of attack is an ideal choice for a reduction in noise emission. The vortex sheet indicates less noise projection when comparing the varied angle of attack to that of the fixed angle of attack. When comparing the wind direction of the fixed angle of attack, the velocity is constant. As the angle of attack increases, the frequency of wake vortex shedding decreases, but the frequency of shear-layer instabilities starts to rise. The azimuth angle enhances streamwise wind, thus improving stall behaviour within the low tip speed ratio range. (2) The impact of the varied angle of attack on the tip speed ratio improves the turbine's power efficiency and torque output. However, the downstream and upstream noise intensifies as the angle of attack increases [21]. As a result, noise prediction at take-off must account for the effect of angles of attack. (3) The loudness and sharpness, which are the metrics that are important for the human perception of sound, are also observed to increase the noise pollution through a varied angle of attack [22]. However, discomfort is associated with the boundary layer tones viewed to be at amplitudes of $60 \mathrm{~dB}$ at frequencies above $1000 \mathrm{~Hz}$ [20]. As a result, the findings of this paper can contribute to a better understanding of noise-generating mechanisms and the design of quieter vertical axis wind turbines.

Furthermore, these findings can be used to establish the most efficient position for wind turbine installation so that the noise emitted is as inconvenient to nearby neighbours as possible. [2]

We want to improve the accuracy of our results with a computational domain that has a higher cell elements well above the million cell count. Then perform the numerical simulation using a computer cluster for parallel computing. We will also perform experiments using signal processing is performed by the beamforming technique to compare numerical results.

\section{Acknowlegdement}

The author would like to thank supervisor Thokozani Justin Kunene and co-supervisor Lagouge Kwanda Tartibu and for the support guidance patience during the preparation of 
this paper. The institution University of Johannesburg for financially committing to the conference cost.

\section{Nomenclature}

A Sweep area $\left[\mathrm{m}^{2}\right]$

c Blade chord length [m]

$\mathrm{C}_{\mathrm{L}} \quad$ Coefficient of Lift

$\mathrm{C}_{\mathrm{D}}$ Coefficient of Drag

$\mathrm{C}_{\mathrm{P}}$ Coefficient of Power

D Turbine diameter $[\mathrm{m}]$

$\mathrm{d} \theta \quad$ Azimuthal increment $\left[{ }^{0}\right]$

$\mathrm{F}_{\mathrm{L}} \quad$ Lift force $[\mathrm{N}]$

$\mathrm{F}_{\mathrm{D}} \quad$ Drag force $[\mathrm{N}]$

$\mathrm{L}$ Length $[\mathrm{m}]$

$H \quad$ Blade height [m]

P Static Pressure $[\mathrm{Pa}]$

Q Torque $[$ N.m]

$R \quad$ Radius of rotor [m]

Re Reynolds number over the blade [-]

$\mathrm{U} \quad$ Wind speed upstream $[\mathrm{m} / \mathrm{s}]$

$\mathrm{v} \quad$ Wind velocity $[\mathrm{m} / \mathrm{s}]$

$V_{\infty} \quad$ Wind speed upstream $[\mathrm{m} / \mathrm{s}]$

\section{Greek}

$\lambda \quad$ Tip Speed Ratio (TSR) [-]

$\rho \quad$ Density of air $\left[\mathrm{kg} / \mathrm{m}^{3}\right]$

$v \quad$ Kinematic Viscosity [m2/s]

$\mu \quad$ Dynamic Viscosity [N.s $\left./ \mathrm{m}^{2}\right]$

$\beta \quad$ Angle-of-Attack [0]

$\omega \quad$ Angular velocity [ $\mathrm{rad} / \mathrm{sec}]$

\section{Subscripts}

geo Geometry of the blade

p Power

\section{Appendix A}

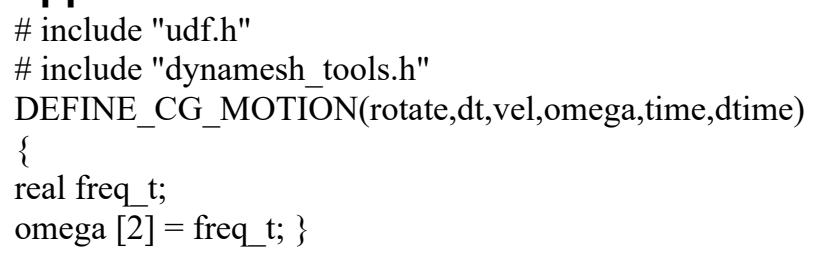

\section{Appendix B:}

\#include "udf.h"

DEFINE_CG_MOTION(yaw, dt, vel, omega, time, dtime)

\{

real a, w, pi;

$\mathrm{pi}=3.1415$;

/* define motion variables */

$\mathrm{a}=0.05 ; / * 0.05 \mathrm{~m}$ movement amplitude */

$\mathrm{w}=2 *$ pi $* 2 ; / * 2 \mathrm{~Hz}$ frequency $* /$

$/ *$ define object movement law $* /$

omega $[0]=-.5 * \sin (\mathrm{w} *$ time $)$;

\}

\section{References}


1. M. Dyrholm, "Global Wind Energy councli, Global Wind Report 2019.2020.," 2019. [Online]. Available: eqmagpro.com/wp-content/upload/2021/03/GWEC-IGlobal-Wind-Report_2021

2. J.N Pinder, "Mechanical noise from wind turbine," Wind Energy, pp. 158-168, 1992

3. Sally Wright, M.S;Anthony L, Rogers, "Renewable Energy Research Laboratory," Massachusetts Amherst, 2006.

4. J. Su et al., "Aerodynamic noise assessment for a vertical axis wind turbine using Improved Delayed Detached Eddy Simulation,” Renew. Energy, vol. 141, pp. 559$569,2019$.

5. CS, Feirreira; G, Van Kuik; G, Van Bussel; F, Scrarano, "Visualization by piv of dynamic stall on vertical axis wind turbine," Exp Fluid, pp. 97-108, 2009.

6. LA, Danao; R, Eboibi OHowell, "An experimental investigation into the influence of unsteady wind on the performance of vertical axis wind turbine," Appl Energy, pp. 107:403-411, 2013.

7. Doolan,CJ;Moreau,DJ;Brooks,LA, "Wind turbine noise mechanism and some concept for its control," Acoust Aaust , pp. 40(1)7-13, 2012.

8. Mohamed, "Aero-acoustic noise evaluation of H-rotor Darius wind turbine," Energy 65, pp. 596-604, 2014.

9. Journal of Mechanical Engineering and Sciences, vol. 1, no. 122, pp. 3399-3409, 2018.

10. Ian Carlo ,M. Lositano; Louis Angelo M. Danao, "Steady wind performance of a $5 \mathrm{KW}$ threee blade H-rotor Darrieus Vertical Axis Wind Turbine with cambered tubercle leading edge blades," Energy 175, pp. 278-291, 2019.

11. K.Brenther and F.Farassat, "Modeling aerodynamically generated sound of helicopter rotors," Aeronaut.SCI 39, pp. 83-12, 2003.

12. J.Ffowcs William;D.Hawking, "Sound generation by turbulence and surfaces in arbitrary motion," Pilos Trans Soc, pp. 321-342, 1969.

13. M.Wang;J.B.Freund;S.K.Lele, "Computational prediction of flow-generated sound," Fluid Mech, pp. 483-512, 2006.

14. F. A.Sunyoto, "The effects of number of blades on the performance of H-Darrieus type wind turbine," QIR Quality in research.IEEE, 2013.

15. R. S.Brusca, "Design of a vertical axis wind turbine:how the aspect ratio effects the turbine's performance," Int J Energy Environ Eng, pp. 5(4):333-40, 2014.

16. A.R.Jha,, Wind turbine techonology., New York: CRC Press, 2011.

17. Al-Shemmer, "Wind turbines.," Wind turbines., vol. 978, no. Al-Shemmeri \& Ventus., pp. 978-87,681-692, 2010.

18. L.Kostic, "Review of the Spalart-Allmaras Turbulence Model and its Modifications to Three-Dimensional Supersonic Configurations," Scientific Technical Review, vol. 1, no. LXV, pp. 43-49, 2015

19. W. S, B. R and G. G, Wind turbine noise, Berlin, 1996.

20. M.Mohamed,G. Janinga,Optimization of Savonius blade using an obstabcle shielding the returning blade. Energy convers management, 52(1)236-42. (2011)

21. Viqueira-Moreira M, Ferrer E. Insights into the Aeroacoustic Noise Generation for Vertical Axis Turbines in Close Proximity. Energies. 2020; 13(16):4148. https://doi.org/10.3390/en13164148 